\title{
Developed approach to determine the seismic vulnerability of reinforced concrete structures
}

\author{
SERRAYE Mahmoud ${ }^{1}$, AMRI Salima ${ }^{2}$ \\ ${ }^{1}$ Geo-materials Laboratory, Civil Engineering Department, Faculty of Technology, Saad Dahleb university of Blida, \\ ALGERIA. \\ ${ }^{2}$ Faculty of Technology, university of Medea, ALGERIA
}

\begin{abstract}
Several evaluation methods of the seismic vulnerability have been developed around the world. Which are very use ful from humanitarian and socioeconomic point of view. Generally these methods use knowledge obtained from previous earthquakes and they are basing on seismic intensity scales and on buildings direct observation. But the macroseismic intensity expresses the consequences of the seism, and not its physical characteristics of the structures. Contrary to this type of methods, an approach based on a nonlinear analysis (Push-Over method) is proposed in this work. It consists in modeling the excitation of the earthquake by a response spectrum and building's behavior by capacity curves. These capacity curves are obtained from numerical modeling performed by Opensees software. The superposition of the two curves, response spectra and capacity curve, makes it possible to determine the performance point and consequently to deduce the state of expected damage. To estimate the probability of damage of a building at a given level of solicitation (defined by $S_{d}$ ), we excites a group of buildings characterized by different parameters related to the geometry of the building and those are related to the materials used (concrete, steel) by seismic solicitation (Response spectrum - RPA 99). The performance point for each building is determined by a procedure defined in FEMA 440. We classifies the buildings according to the position of performance point on their curve which defines a damage state of ds (Mild, Moderate, Important or Ruin) according to the damage levels of Risk-UE. A statistical analysis is then made for each class to build the fragility curves.
\end{abstract}

\section{INTRODUCTION}

Aujourd'hui, nombre important des modèles d'estimation de dommages sismiques ont pu être réalisés à travers le monde, permettant la réalisation des scénarii catastrophe, très utiles d'un point de vue humanitaire, social ou économique pour la gestion d'une éventuelle crise. Généralement les méthodes d'évaluation de la vulnérabilité sismiques actuelles correspondent à des approches basées sur l'utilisation des relations entre intensité macrosismique et les dommages post sismiques, telle que l'EMS 98, GNDT, RISK-UE "level I"... Mais la valeur de l'intensité macrosismique exprime les conséquences du séisme, et fonction de l'ampleur des dommages.

Contrairement à ce type des méthodes, l'estimation de l'état de dommages sur la base du comportement du bâtiment (courbes de capacité) prend directement en compte les paramètres liés au mouvement du sol, tels que l'accélération et le déplacement, en plus le comportement (réponse) des structures dépend fortement avec les dimensions géométries des constructions (hauteur d'étage, nombre d'étages, nombre portées aux deux directions et taille...). L'approche HAZUS représente la seule méthode présentant à ce jour une approche de ce type, son caractère exhaustif et notamment l'explicitation des relations utilisées ont imposé ce modèle comme une référence au niveau international, mais son accessibilité uniquement aux spécialistes, influer à son utilisation, reste limité (moins publique). Le projet européen RISKUE "level II" a comme objectif le développement un modèle spécifique au contexte européen. C'est cette caractéristique de méthodes existantes qui a poussé la communauté scientifique à développer une approche s'appuyant sur la méthode de capacité spectrale (capacity spectrum method of ATC-40, 1996) [1].

Cette approche a été développée dans cette communication, basée sur des courbes de capacités spécifiques au contexte du bâti urbain algérien. Les courbes de capacités ont la propriété d'exprimer un niveau d'endommagement directement en fonction de la sollicitation sismique spécifique (spectre de réponse RPA99) à l'enjeu étudié. Dans ce qui suit les différentes étapes de l'approche développée.

\section{PROCEDURE L'APPROCHE DEVELOPPEE}

L'approche développée pour l'estimation de dommages repose sur la méthode push-over, elle se décompose en quatre étapes principales: 


\subsection{La première étape: construction les courbes de capacité}

Une analyse non linéaire (analyse push over) des structures à l'aide une modélisation numérique est réalisée par logiciel Opensees pour construire les courbes de capacité. Les courbes tirant en terme effort tranchant (V) à la base en fonction du déplacement (D) au sommet. Ces courbes sont transformées en termes d'accélération spectrale ' $S a$ ' et de déplacement spectral ' $S d$ ' (voir figure 01et 02) par les relations [1] suivantes:

$$
\begin{gathered}
S_{d}=\frac{D}{I_{1} \Phi_{t-1}} \\
S_{\sigma}=\frac{V / W}{}
\end{gathered}
$$

$$
\alpha \text { s }
$$

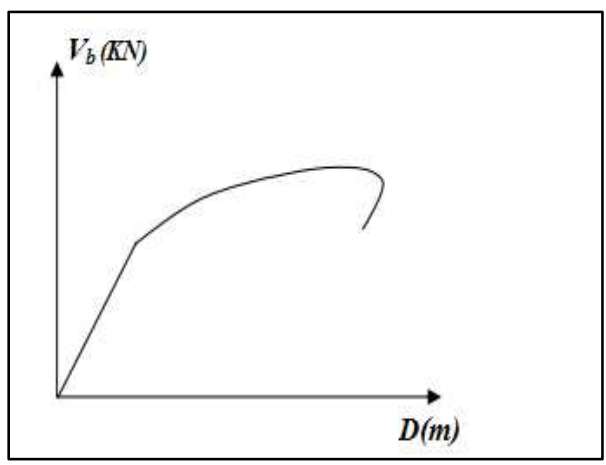

Fig. 1. La courbe de capacité au format V-D

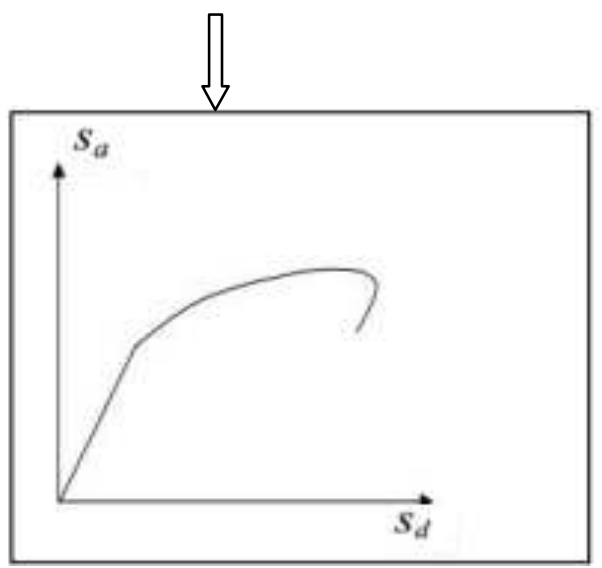

Fig. 2. La courbe de capacité au format $\mathrm{Sa}-\mathrm{Sd}$

Comme recommandé dans le document ATC40 [1], on adopte pour les actions sismiques appliquées à la structure de forme triangulaire et en supposant que déformée modale est normalisée à $\quad \Gamma_{1} \phi_{t, 1}=1.4$, et les facteurs de charges appliquer lors d'une analyse PushOver $\alpha=0.8[1]$.

Avant la modélisation numérique elle consiste de caractériser les bâtiments étudies selon une classe typologique définie par des paramètres lié à la géométrie du structure:

- Nombres et hauteur d'étage,

- Nombres et longueur de travées en deux sens zz et xx,

- Section des poteaux,
Et par des paramètres lié aux matériaux (béton, acier) telle que :

- La résistance à la compression de béton (fc).

- Limite élastique de l'acier (fy).

Dans cette étape, nous avons générés les paramètres des structures par la simulation de Monte-Carlo.

\subsection{La seconde étape : Choix de l'action sismique}

Dans cette approche, la sollicitation du séisme est représentée par un spectre de réponse la norme RPA99 version 2003 [2] transformé en terme d'accélération spectrale $\mathrm{Sa}$, et déplacement spectral $\mathrm{Sd}$, (voir figure 3) selon les formules suivantes [1] :

$$
\begin{aligned}
& S_{a i}=\left(\frac{4 \pi^{2}}{T_{i}^{2}} S_{d i}\right) / g \\
& S_{d i}=\left(\frac{T_{i}^{2}}{4 \pi^{2}} S_{a i}\right)
\end{aligned}
$$

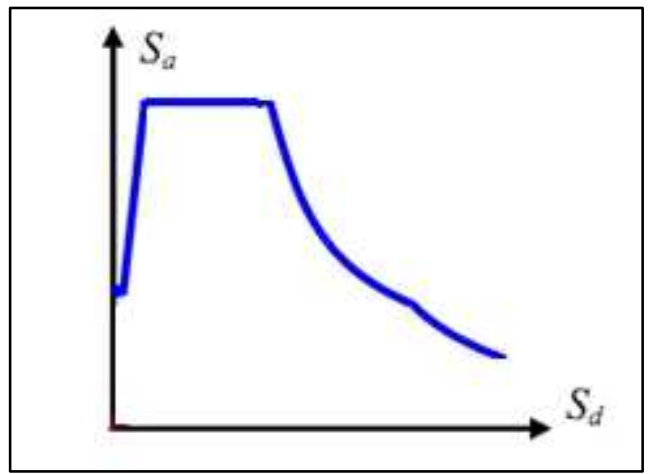

Fig. 3. Définition de l'action sismique.

Nous avons tenu compte des variabilités aux actions sismiques (spectre de réponse) est intégrée au effet de site S [2] et le facteur de qualité Q, (voir figure 04)

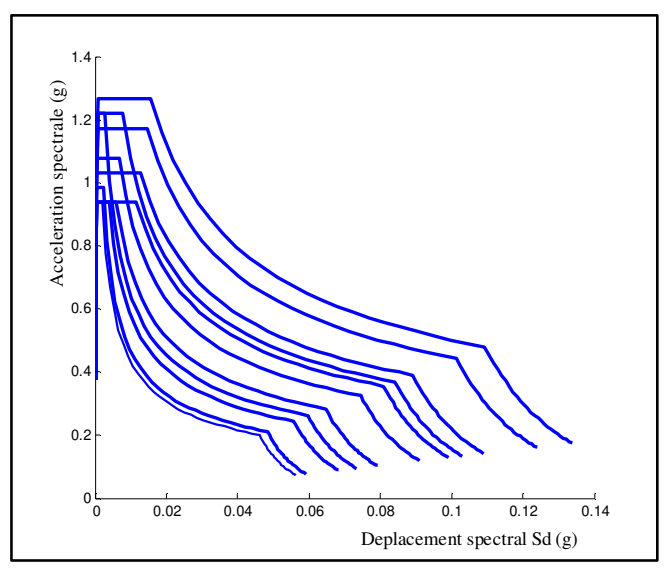

Fig. 4. Variabilités des spectres de réponse. 


\subsection{Troisième étape : Obtention des points de performance}

Ils existent plusieurs techniques pour obtenir la valeur de point de performance de structure. Dans l'approche développée, les coordonnées des points de performance sont calculés à travers la superposition de la courbe de spectre de réponse au format ADRS et les courbes de capacité transformées au format ( $\mathrm{Sa}, \mathrm{Sd}$ ) par une procédure itérative présentée au FEMA 440 (2009) [4].

En fonction la position le point de performance sur la courbe de capacité détermine un certain niveau de dommage. Ainsi, plusieurs niveaux d'endommagement peuvent être distingués à travers cette représentation graphique.

position point de performance

Exemple de

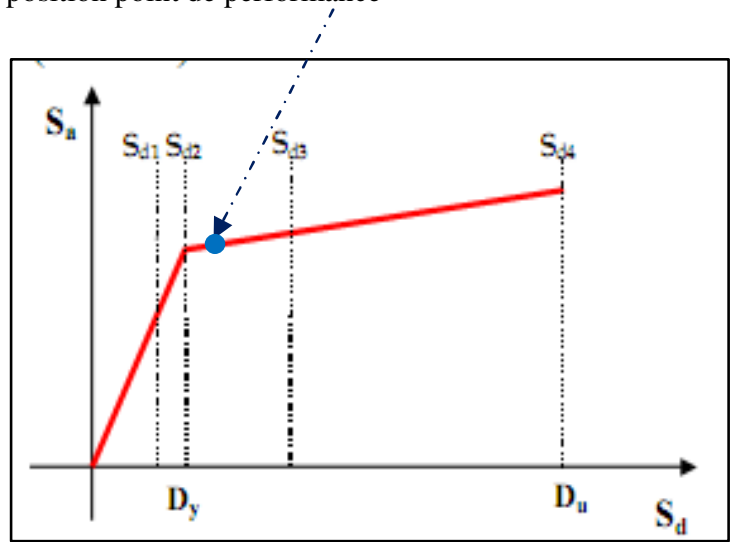

Fig. 5. Signification la position de performance sur la courbe de capacité.

Dans cet approche, on va utiliser les niveaux d'endommagement fournies par programme Risk-UE [3] qui définie quatre niveaux ou degrés de dégâts: faibles, modérés, importants et très importants. Les niveaux d'endommagements proposés par le projet Risk-UE sont présentés au Tableau 01 [3].

Tableau .1 Définitions des niveaux d'endommagement selon Risk-UE [3].

\begin{tabular}{|c|l|c|}
\hline & \multicolumn{1}{|c|}{ Definition } & $\begin{array}{c}\text { Spectral Displacement } \\
\text { Limits (UNIGE, } \\
\text { CIMNE) }\end{array}$ \\
\hline $\mathbf{0}$ & No damage & $\mathbf{D}<0.7$ Dy \\
\hline $\mathbf{1}$ & Slight damage & $0.7 \mathrm{Dy} \leq \mathbf{D}<1.0$ Dy \\
\hline $\mathbf{2}$ & Moderate damage & 1.0 Dy $\leq \mathbf{D}<\mathrm{Dy}+$ Duy \\
\hline $\mathbf{3}$ & Extensive damage & Dy + Duy $\leq \mathbf{D}<$ Du \\
\hline $\mathbf{4}$ & Very heavy damage & Du $\leq \mathbf{D}$ \\
\hline & & Duy $=0.25^{*}($ Du-Dy) \\
\hline
\end{tabular}

\subsection{Quatrième étape : Construction des courbes de fragilité}

Dans ce travail le modèle de distribution statistique retenu pour représenter les fonctions de fragilité de la structure est un modèle de type Log-normal qui représente convenablement la combinaison de variables dont les effets ont multiplicatifs (Chintanapakdee et Chopra, 2003). Cette fonction est contrôlée par deux paramètres: la valeur moyenne $\mathrm{Sd}$ et son écart type $\beta_{\mathrm{Sd}}$. Sa densité de probabilité cumulée s'exprime par la relation suivante [5]:

$$
P\left[d s \mid S_{d}\right]=\Phi\left[\frac{1}{\beta_{d s}} \ln \left(\frac{S_{d}}{\bar{S}_{d, d s}}\right)\right]
$$

$S_{d, d s}$ est la valeur médiane du déplacement spectral pour laquelle la structure atteint le seuil de l'état de dommages ds ; $\beta_{d \mathrm{~s}}$ est l'écart type du déplacement spectral pour l'état de dommages ds, et $\Phi$ est la fonction de répartition de la loi normale centrée réduite. La valeur médiane du déplacement spectral $\mathrm{Sd}$ est déterminée pour chaque niveau de dommage ds à partir des déformations inter-étages moyennes $\Delta$ ds est calculé par l'expression [1] :

$$
S_{d, d s}=\frac{\Delta_{d s} \times H}{\Gamma_{1}}
$$

Avec $\mathrm{H}$ hauteur de la structure et $\Gamma 1$ facteur de participation du premier mode, selon la forme des déformations du structures, le document ATC40 donne des valeurs simplifiés pour $\Gamma 1$, sur cette base en supposant que $\Gamma 1=1.4$ [1] qui correspondant notre cas.

\section{EXEMPLE D'APPLICATION ET RESULTATS OBTENUES}

Dans cette étude, nous nous intéressons à une seule typologie, c'est-à-dire nous avons fait le choix de traiter les constructions en béton armé type portique «poutrespoteaux». Ce choix de typologie est basé sur l'importance et la prépondérance de ces constructions dans le milieu urbain Algérien. Les structures étudiés ont été différenciées en fonction des paramètres liés aux géométries des structures, tel que le nombre d'étages "low-Rise" (entre 1 et 3 étages) ainsi que nombre de travée. Etc. des paramètres liés aux matériaux utilisés de la construction (béton, acier). 


\subsection{Construire les courbes de capacité}

Les 1000 bâtiments étudies de classe typologique "lowRise" (entre 1et 3 étages) et sont différenciées par caractères liés à la géométrie de structure (hauteur d'étage, nombre d'étages, nombre trames et section des poteaux ...) et par des paramètres liés aux matériaux de construction.

Le tableau 2. Donne les différents paramètres lié à la géométrie de la structure et paramétrés lié au matériau de construction.

\begin{tabular}{|c|c|c|c|}
\hline & Paramètre & Nombre d & bâtiments \\
\hline \multirow{19}{*}{ 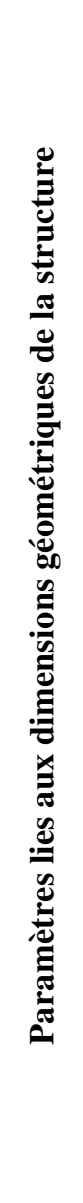 } & \multirow{3}{*}{ Nombres d'étages } & $R$ & 130 \\
\hline & & $R+1$ & 800 \\
\hline & & $R+2$ & 70 \\
\hline & \multirow{3}{*}{$\begin{array}{l}\text { Hauteur d'étage } \\
(\mathrm{m})\end{array}$} & Moyenne & Ecart type \\
\hline & & 3.20 & 0.20 \\
\hline & & \multicolumn{2}{|c|}{ Nombre des bâtiments } \\
\hline & \multirow{3}{*}{$\begin{array}{c}\text { Nombre de travée } \\
z z\end{array}$} & 2 & 70 \\
\hline & & 3 & 180 \\
\hline & & 4 & 750 \\
\hline & \multirow{2}{*}{$\begin{array}{l}\text { 4) Longueur de } \\
\text { trames } z z(m)\end{array}$} & Moyenne & Ecart type \\
\hline & & 4.00 & 0.20 \\
\hline & & \multicolumn{2}{|c|}{ Nombre des bâtiments } \\
\hline & \multirow{3}{*}{$\begin{array}{l}\text { 5) Nombre de } \\
\text { travée } x x\end{array}$} & 2 & 55 \\
\hline & & 3 & 820 \\
\hline & & 4 & 125 \\
\hline & \multirow{2}{*}{$\begin{array}{l}\text { 6) Longueur de } \\
\text { trames } x x(m)\end{array}$} & Moyenne & Ecart type \\
\hline & & 4.00 & 0.20 \\
\hline & \multirow{2}{*}{$\begin{array}{l}\text { 7) Section des } \\
\text { poteaux }(\mathrm{cm})\end{array}$} & Moyenne) & Ecart type \\
\hline & & $30 \times 30$ & 5.00 \\
\hline \multirow{4}{*}{ 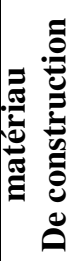 } & \multirow{2}{*}{$\begin{array}{l}\text { La résistance à la } \\
\text { compression de } \\
\text { béton }(f c)(M p a)\end{array}$} & Moyenne & Ecart type \\
\hline & & 20.00 & 3.25 \\
\hline & \multirow{2}{*}{$\begin{array}{l}\text { Limite élastique } \\
\text { de l'acier fy } \\
\qquad(\text { (Mpa) }\end{array}$} & Moyenne & Ecart type \\
\hline & & 420.00 & 20.00 \\
\hline
\end{tabular}

Nous présentons les différentes courbes de capacité de bâtiments étudiés dans notre travail à la figure 06 .

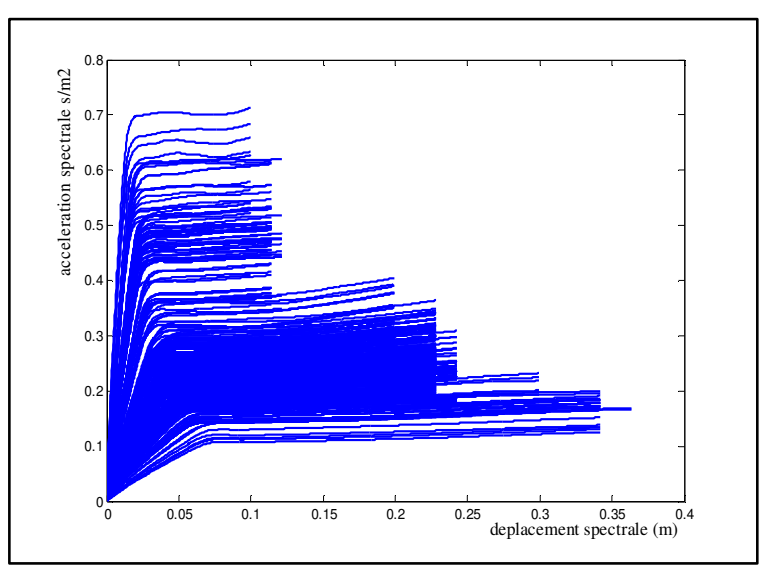

Fig. 6. Courbes de capacité en format ADRS (exemple de 1000 bâtiments).

\subsection{Générer les spectres de réponse}

Dans l'approches développée, nous allons utiliser une sollicitation sismique est considérée à travers un spectre de réponse de Règlement Parasismique Algérienne (RPA 99 version 2003) [37] qui définie par:

- la zone sismique III.

- le groupe d'usage groupe1B $(A=0.30)$.

32 combinaisons sont formés par des différentes valeurs de facteur de qualité $\mathrm{Q}(1,1.05,1.10,1.15,1.20,1.25$, $1.35)$ avec le site $\mathrm{T} 2(0.30,0.40,0.50,0.70)$ permettant de générés le spectre de réponse dans le plan $(\mathrm{Sa}-\mathrm{Sd})$ comme présenté à la figure 07 .

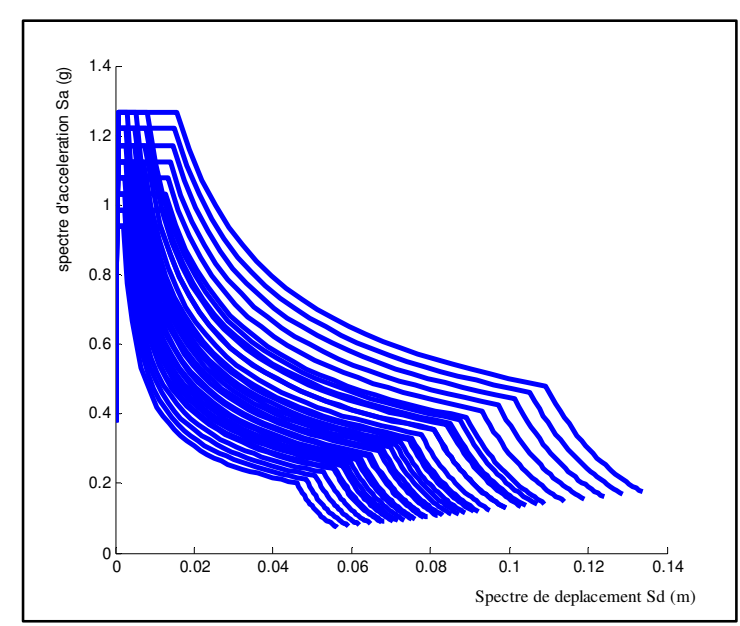

Fig. 7. Génération spectres de réponse (32 combinaisons entre $\mathrm{Q}$ et $\mathrm{T} 2$ ).

\subsection{Déterminer les points de performances}

Dans le présent de travail, on utilise une procédure itérative définie par le code FEMA440 (2009) pour la détermination les points de performance comme présentée aux figures 08 . Nous avons considéré dans cet exemple 1000 bâtiments (courbes de capacité) soumise 
aux différentes agressions sismiques (32 spectre de réponse) (voir la figure 09 ).

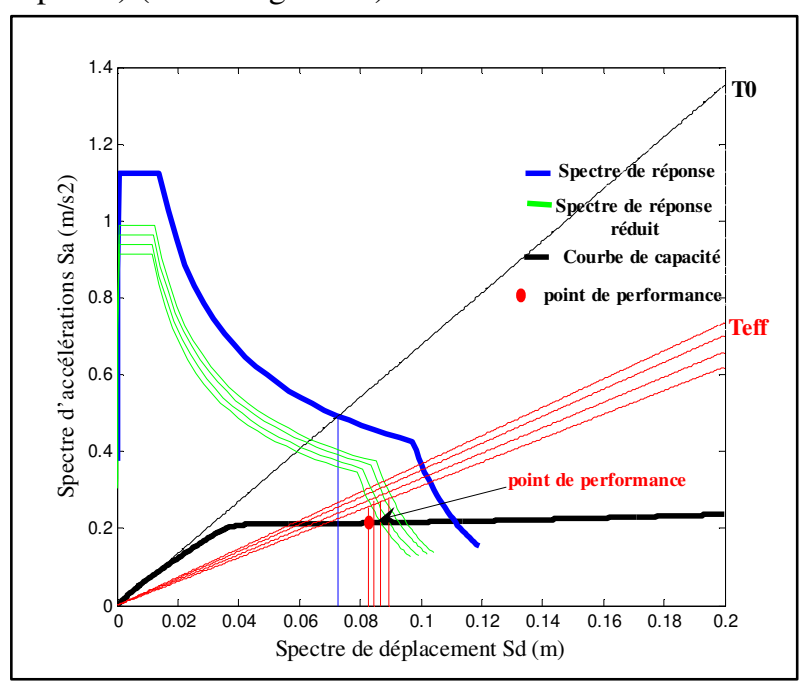

Fig. 8. Schéma pour d'obtention du point de performance selon procédure « FEMA440 ».

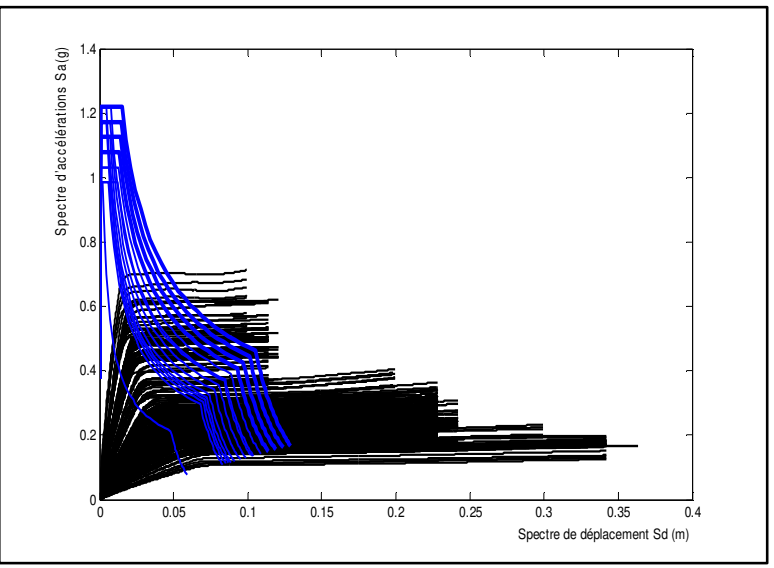

Fig. 9. Superposition entre courbes de capacité (1000 bâtiments) et spectres de réponse générée par 32 combinaisons.

\subsection{Construire des courbes de fragilité}

D'après la classification des bâtiments selon l'état d'endommagent (faibles, modérés, importants et très importants). On détermine la valeur moyenne de $S_{d}$ et l'écart type de chaque classe ds. Une fois les paramètres de la fonction de fragilité $\mathrm{Sd}$,ds obtenus, on peut construire les courbes de fragilité à partir de l'équation (04). Les courbes de fragilité sont représentées à la figure 10 .

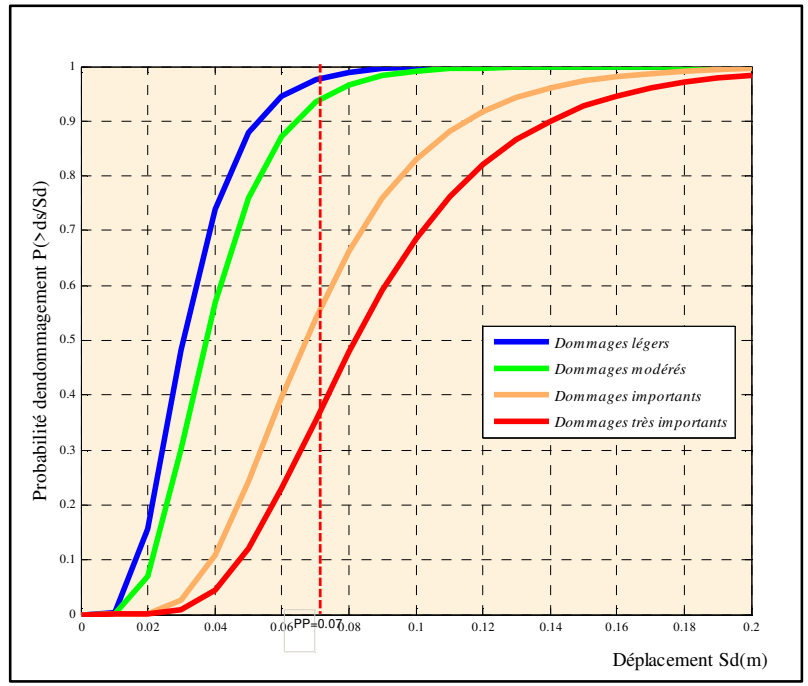

Fig. 10.Construction courbes de fragilité.

Connaissant le déplacement spectral maximum $\mathrm{Sd}$ il est possible de déterminer la probabilité d'endommagement, en fonction des quatre niveaux de dommages. Ainsi, pour un déplacement subi de $\mathrm{X} \mathrm{cm}$, nous pouvons quantifier les probabilités d'avoir respectivement P1\% d'endommagement de niveau 1 (dommages légers), P2\% d'endommagement du niveau 2 (dommages modérés), P3\% d'endommagement du niveau 3 (dommages importants), P4\% d'endommagement du niveau 4 (dommages très importants allant jusqu'à la ruine). A titre illustratif, pour un déplacement spectral de $7 \mathrm{~cm}$, les probabilités que la structure se trouve aux niveaux d'endommagement $1,2,3$ ou 4 sont respectivement de $97 \%, 93 \%, 55 \%$ et $37 \%$.

\section{CONCLUSION}

Par cette communication nous avons essayé de contribuer au développement d'un outil (courbes de fragilité) permettant l'amélioration de la gestion du risque sismique en Algérie.

Une méthodologie développée pour la détermination des courbes de fragilité a été présentée. Cette méthodologie est basée sur de la méthode push-over et l'utilisation des simulations Monte Carlo. Des courbes de fragilité ont été établies pour des structures de type portique en béton peuvent être utilisées pour la mise en conformité sismiques de structures existantes. Les résultats (courbes de fragilité) présentés ici constituent un exemple d'utilisation pour l'évaluation des dommages et constituent une première estimation des niveaux de risques dans la définition du risque sismique des constructions. Les perspectives directes de ce travail, sont l'application de la méthodologie développée et sa validation sur d'autres typologies de structures

De nombreux études de recherches dans le risque naturel et notamment sismique se sont réalisés dans les dernières années (HAZUS, RISK-EU). Ils ont apporté ainsi une quantité importante d'information, concernant essentiellement le développement de nouvelles solutions techniques pour les constructions et la protection de 
différents enjeux d'une part, et l'estimation des conséquences potentielles suite à la manifestation des différents scénarii de catastrophes naturelles d'autre part. Les décideurs disposent par conséquent aujourd'hui d'une large gamme d'outils, dont certains présentés dans ce travail, qui doivent servir comme un appui pour la mise en place d'une meilleure politique de mitigation. Les résultats obtenus nous semblent cohérents avec ceux déjà obtenus par d'autres chercheurs, Tout fois nous devons procéder à une validation de ces résultats en les comparant à des endommagements enregistrés lors de séisme (exemple ceux de Boumerdes).

\section{REFERENCES}

[1] Applied Technology Council ATC.40, (1996).

[2] Règlement Parasismique Algérienne RPA $(99$ version 2003).

[3] RISK-UE, An advanced approach to earthquake risk scenarios with applications to different european towns, Projet Européen, EVK4-CT-2000-00014, (2003).

[4] Federal Emergency Management Agency FEMA440,(2009).

[5] HAZUS. Direct physical damage general building stock, chapter 5, HAZUS99 Technical Manual, (1999). 